\title{
Full-Wave Spectral-Domain Analysis of Compensation of Microstrip Discontinuities Using Triangular Subdomain Functions
}

\author{
Tzyy-Sheng Horng, Member, IEEE, William E. McKinzie, and Nicolaos G. Alexopoulos, Fellow, IEEE
}

\begin{abstract}
This paper presents a full-wave spectral-domain analysis to investigate compensation of a variety of microstrip discontinuities including open-ends, bends and $T$ junctions. To properly model the discontinuities with miters as well as $90^{\circ}$ corners, vector-valued triangular subdomain functions are used as both expansion and testing functions in the moment method procedure. Special consideration is given to the numerical treatment of the reaction integral between two triangular subdomains such that rather complicated geometrical configurations can be handled very efficiently. Comparison of some numerical results with available experimental data shows excellent agreement. The losses due to radiation and surface waves for some discontinuities are also included.
\end{abstract}

\section{INTRODUCTION}

$\mathrm{I}^{\mathrm{N}}$ $\mathrm{N}$ THE design of microwave and millimeter wave circuits, compensation of microstrip discontinuities is widely used to reduce the effects of discontinuity reactances [1], [2]. For low frequency applications, planar waveguide models have been successfully applied for compensation of some discontinuities such as steps, rightangle bends and $\mathrm{T}$ junctions [3], [4]. At higher frequencies, a dynamic model based on a full-wave analysis is required to take into account more physical effects such as radiation and surface-wave losses. The most rigorous full-wave method for the characterization of open microstrip discontinuities is governed by the well-known electric field integral equation (EFIE), which can be formulated in both the space domain [5], [6] and spectral domain [7]-[11]. In the space domain, the dyadic kernel in the EFIE is the Green's function for the electric field which can be obtained from a Sommerfeld-type integral. Since the kernel is highly singular, the evaluation of the reaction integrals in the moment method procedure is difficult when the observation point is within the integration range. The mixed potential integral equation (MPIE), which is a modification of the EFIE, is usually solved in the space domain [12], [13]. In comparison with the EFIE in the space domain, the kernel in the MPIE is less singular,

Manuscript received March 31, 1992; revised August 3, 1992. Th1s research was supported by National Science Foundation Research Grant ECS 8802617.

The authors are with the Electrical Engineering Department, University of California, Los Angeles, Los Angeles, CA 90024.

IEEE Log Number 9203693. which makes the evaluation of reaction integrals more numerically simple and stable for two near-by elements.

The spectral-domain analysis performs an integral transformation, usually Fourier or Hankel transforms, to transform a partial differential equation into an ordinary differential equation. After satisfying the boundary conditions at the interfaces of multi-layer stratified dielectric medium, this approach can lead to a closed-form expression for the so called spectral-domain dyadic Green's function. The space-domain electromagnetic fields can be expressed as the inverse Fourier transform of the vector product of this spectral-domain dyadic Green's function and Fourier transform of the microstrip currents. Such an EFIE formulation in the spectral domain has several advantages over the other two space-domain analyses. One advantage is that the integration path may be chosen to avoid singularities, thus yielding a smoothly varying integrand. A second advantage is the opportunity to independently determine both radiation and surface-wave losses [14]. One can compute the impedance matrix in the method of moments very accurately using the spectraldomain formulation such that the circuit parameters in a microstrip junction can be rigorously determined.

A review of past work using full-wave spectral-domain analysis for open microstrip discontinuities reveals that the technique cleveloped up to now is limited to a multiport junction whose shape can be divided into a number of rectangles. The most commonly used expansion functions, pulse and piecewise sinusoidal functions, are appropriate only for modeling a discontinuity with $90^{\circ}$ corners. To model the discontinuities with miters at any angle, vector-valued triangular subdomain functions are adopted in this analysis as both expansion and testing functions in the moment method procedure. These triangular subdomain functions were apparently first employed by Rao, Wilton and Glisson [15]. They are suitable for modeling electric currents on arbitrary PEC surfaces. However, the present application will restrict the surface patches to lie in the plane of discontinuities. Since a spectral-domain approach is employed in the characterization of microstrip discontinuities, the Fourier transform of the triangular subdomain function has to be derived. This results in a very complicated mathematical expression. Crucial to the numerical formulation is the efficient compu- 
tation of the reaction integrals associated with these triangular subdomains to avoid tremendous computation time. An efficient method to improve numerical efficiency is to employ a space-domain technique for the asymptotic solution of the spectral-domain reaction integral [16], [17].

\section{SPectral-Domain Matrix Formulation}

\section{A. Electric Field Integral Equation}

Consider a microstrip structure where the electric current distribution is viewed in terms of a number of infinitesimal dipoles continuously distributed on the interface between the dielectric and the air. From linear superposition, the tangential electric field on the interface can be expressed by a two-dimensional spatial convolution of the dyadic Green's function with the current distribution. Each component of this two-dimensional spatial convolution corresponds to multiplication of the two-dimensional Fourier transforms in the spectral domain. Therefore, the space-domain tangential electric field can be expressed as an inverse Fourier transform of the vector product of the spectral-domain dyadic Green's function and the Fourier transform of the microstrip currents. An EFIE can be obtained by imposing the boundary condition that the total tangential field is zero on the conductors:

$$
\begin{gathered}
\vec{E}_{t}(x, y)=\frac{1}{4 \pi^{2}} \int_{-\infty}^{\infty} \int_{-\infty}^{\infty} \overline{\bar{G}}\left(k_{x}, k_{y}\right) \cdot \overrightarrow{\tilde{J}}\left(k_{x}, k_{y}\right) e^{-j k_{x} x} \\
\cdot e^{-j k_{y y}} d k_{x} d k_{y}+\vec{E}^{i n c}(x, y)=0 \\
\text { for } x, y \text { on microstrips }
\end{gathered}
$$

where $\overline{\bar{G}}$ and $\overrightarrow{\tilde{J}}$ are the spectral-domain dyadic Green's function and the current distribution, respectively. The closed-form expression of the spectral-domain dyadic Green's function [18] takes into account all the physical phenomena including radiation and surface waves. $\vec{E}^{i n c}$ is an impressed electric field used to model a voltage source on a microstripline.

\section{B. Triangular Subdomain Functions}

The triangular subdomain functions as shown in Fig. 1 are vector-valued functions defined as

$$
\begin{aligned}
& \vec{f}_{n}(x, y) \\
& =\left\{\begin{array}{c}
\frac{l_{n}}{2 A_{n}^{+}}\left[\left(x-x_{3}\right) \hat{x}+\left(y-y_{3}\right) \hat{y}\right], \\
x, y \text { in } T_{n}^{+} \\
\frac{l_{n}}{2 A_{n}^{-}}\left[\left(x_{4}-x\right) \hat{x}+\left(y_{4}-y\right) \hat{y}\right], \\
x, y \text { in } T_{n}^{-} \\
0, \quad \text { otherwise }
\end{array}\right.
\end{aligned}
$$

where $T_{n}^{+}$and $T_{n}^{-}$denote the domains of two adjacent triangles with areas $A_{n}^{+}$and $A_{n}^{-}$respectively. Fig. 1 also

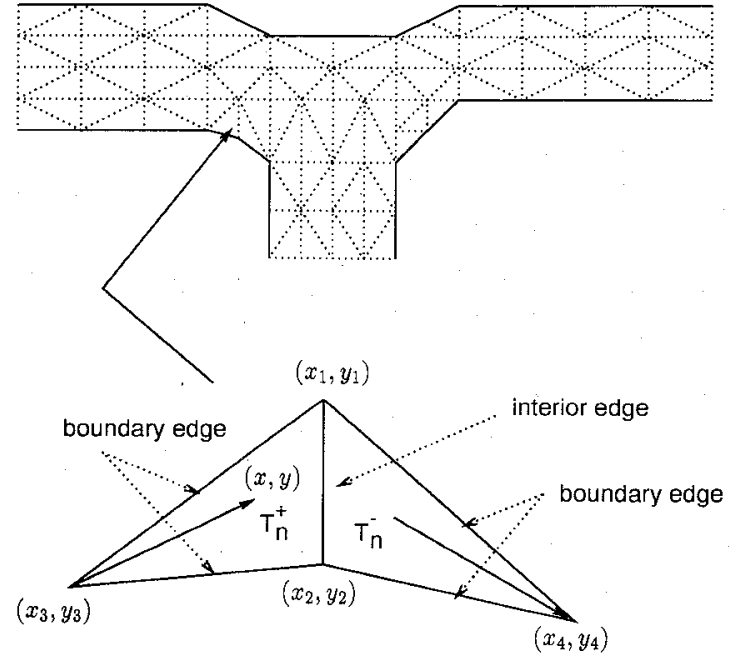

Fig. 1. Triangular subdomain function.

shows a microstrip discontinuity of arbitrary shape on a substrate with permittivity $\epsilon_{r}$ and substrate thickness $h$. The discontinuity is inscribed with non-overlapping triangles defined in terms of an appropriate set of vertices, faces, interior edges and boundary edges. The $n$th expansion function $\left(\vec{f}_{n}\right)$ is uniquely associated with a pair of adjacent triangles sharing a common edge (interior edge) whose length is $l_{n}$. The current on microstrip may be expanded as

$$
\vec{J}(x, y) \simeq \sum_{n=1}^{N} I_{n} \vec{f}_{n}(x, y)
$$

where $N$ is the total number of the interior edges and $I_{n}$ are unknown coefficients to be obtained through the method of moments. This basis function has two salient properties which make it uniquely suited to approximate electric surface currents on microstrip discontinuities of arbitrary shape [15]:

1. The current has no component normal to the boundary edge.

2. The component of current normal to the $n$th interior edge is constant and continuous across the edge.

This triangular subdomain function basically consists of a pair of linearly varying functions with triangular support whose Fourier transform has been derived recently in [19], [20]. In this paper, we define the Fourier transform of a triangular subdomain function as

$$
\overrightarrow{\tilde{f}}_{n}\left(k_{x}, k_{y}\right) \equiv \int_{-\infty}^{\infty} \int_{-\infty}^{\infty} \vec{f}_{n}(x, y) e^{j k_{x} x} e^{j k_{y y} y} d x d y
$$

\section{Galerkin's Procedure}

After substituting the expanded current expression (3) and the Fourier transform of expansion functions (4) into (1), the method of moments can be applied to convert this integral equation into a matrix equation. The testing functions in the moment method procedure are also chosen to be triangular subdomain functions. As a result, the matrix 
equation for a microstrip discontinuity is generally formulated as

$$
[Z]_{N \times N}[I]_{N}=[V]_{N} .
$$

Each matrix element in $[Z]$ represents the reaction between two basis functions. The computation of each element requires a double infinite integration in the spectral domain:

$$
\begin{aligned}
Z_{m n}= & \frac{1}{4 \pi^{2}} \int_{-\infty}^{\infty}\left[\overline{\bar{G}}\left(k_{x}, k_{y}\right) \cdot \overrightarrow{\tilde{f}}_{n}\left(k_{z}, k_{y}\right)\right] \\
& \cdot \overrightarrow{\tilde{f}}_{m}^{*}\left(k_{x}, k_{y}\right) d k_{x} d k_{y}
\end{aligned}
$$

where the superscript $*$ represents the complex conjugate of the function. In (5), the right-hand side matrix represents an excitation mechanism for the microstrip junction. In this analysis, an ideal delta-gap voltage source is placed near the end of a finite length of microstripline to excite the dominant mode. We can think of this source as a concentrated slot electric field where the slot or gap is cut along the edges of the triangular patches. The detailed mathematical formulation of the excitation elements can be found in [21].

For general applications, each microstrip junction is assumed to be connected to finite lengths of microstriplines. Triangular subdomain functions are used exclusively to expand the electric surface current density on the junction as well as the microstriplines. Once the current distribution along the microstriplines is obtained from the solution of the matrix equation, the scattering parameters of the junction can be further extracted from knowledge of this current distribution and the propagation constant of the dominant mode [6]. Empirically, each microstripline needs to be longer than a half guided wavelength to yield accurate results.

\section{Numerical Evaluation of Matrix Elements}

\section{A. Integration in Polar Coordinates}

After transforming into polar coordinates, the double infinite integration in (6) is carried out numerically. We reduce the integration to a finite integral $(0$ to $2 \pi)$ with respect to an angular variable $\theta=\tan ^{-1}\left(k_{y} / k_{x}\right)$ and an infinite integral $(0$ to $\infty)$ with respect to a radial variable $\lambda=\sqrt{k_{x}^{2}+k_{y}^{2}}$. For the finite integral; since the integrand is well behaved over the angular domain, a 16 to 32 point
Gaussian quadrature formula can be used depending on the distance between the expansion and testing functions. For the infinite integral, the integrand containing the Fourier transforms of the triangular subdomain expansion and testing functions is mathematically complex and converges slowly. Computation of impedance matrix elements involving triangular subdomains takes approximately 4 times the CPU time when compared to the integrand using pulse or piecewise sinusoidal functions in modeling the discontinuities with $90^{\circ}$ corners. An efficient method to perform the integration is to apply an asymptotic extraction technique [16]. This technique breaks the double infinite integral into two integrals. One is the asymptotic integral (denoted by $Z_{m n}^{a}$ ) where the leading term of the asymptotic expansion of $\overline{\bar{G}}$ as $\lambda \rightarrow \infty$ is used in the integrand. The other is the difference integral (denoted by $Z_{m n}^{d}$ ) where subtraction of this leading term from $\overline{\widetilde{G}}$ is used in the integrand. After a slight arrangement, it is found that the leading term of the asymptotic expansion of $\overline{\bar{G}}$ is asymptotically equivalent to the dyadic Green's function in a homogeneous medium with the dielectric constant equal to the average of the dielectric constants immediately above and below the source points. As a result, both integrals can be mathematically expressed as follows:

$$
\begin{aligned}
& Z_{m n}=Z_{m n}^{a}+Z_{m n}^{d}, \\
& Z_{m n}^{a}=\int_{0}^{\infty} A_{m n}(\lambda) d \lambda, \\
& Z_{m n}^{d}=\int_{0}^{\infty} D_{m n}(\lambda) d \lambda,
\end{aligned}
$$

where

$$
\begin{aligned}
A_{m n}(\lambda)= & \frac{\lambda}{4 \pi^{2}} \int_{0}^{2 \pi}\left[\overline{\overline{\tilde{G}}}_{a}(\lambda, \theta) \cdot \overrightarrow{\tilde{f}}_{n}(\lambda, \theta)\right] \\
& \cdot \overrightarrow{\tilde{f}}_{m m}^{*}(\lambda, \theta) d \theta, \\
D_{m n}(\lambda)= & \frac{\lambda}{4 \pi^{2}} \int_{0}^{2 \pi}\left[\left\{\overline{\bar{G}}(\lambda, \theta)-\overline{\overline{\tilde{G}}}_{a}(\lambda, \theta)\right\}\right. \\
& \left.\cdot \overrightarrow{\tilde{f}}_{n}(\lambda, \theta)\right] \cdot \overrightarrow{\tilde{f}}_{m m}^{*}(\lambda, \theta) d \theta,
\end{aligned}
$$

$$
\begin{aligned}
\overline{\overline{\tilde{G}}}\left(k_{x}, k_{y}\right)= & \frac{-j \omega \mu_{0}}{k_{0}^{2}} \frac{1}{\lambda_{0}+\lambda_{\epsilon_{r}} \operatorname{coth} \lambda_{\epsilon} h}\left[\begin{array}{cc}
k_{0}^{2}-k_{x}^{2} & -k_{x} k_{y} \\
-k_{x} k_{y} & k_{0}^{2}-k_{y}^{2}
\end{array}\right] \\
& +\frac{-j \omega \mu_{0}}{k_{0}^{2}} \frac{\lambda_{0}\left(\epsilon_{r}-1\right)}{\left(\lambda_{0}+\lambda_{\epsilon_{r}} \operatorname{coth} \lambda_{\epsilon_{r}} h\right)\left(\epsilon_{r} \lambda_{0}+\lambda_{\epsilon r} \tanh \lambda_{\epsilon} h\right)}\left[\begin{array}{ll}
k_{x}^{2} & k_{x} k_{y} \\
k_{x} k_{y} & k_{y}^{2}
\end{array}\right] \\
& \sim \frac{-j \omega \mu_{0}}{k_{0}^{2}} \frac{1}{2 \lambda_{h} \epsilon_{h}}\left[\begin{array}{cc}
k_{h}^{2}-k_{x}^{2} & -k_{x} k_{y} \\
-k_{x} k_{y} & k_{h}^{2}-k_{y}^{2}
\end{array}\right] \\
= & \overline{\bar{G}}_{a}\left(k_{x}, k_{y}\right) ; \lambda \rightarrow \infty
\end{aligned}
$$




$$
\begin{aligned}
\epsilon_{h} & =\frac{\epsilon_{r}+1}{2}, \\
k_{0} & =\omega \sqrt{\mu_{0} \epsilon_{0}}, \\
k_{h} & =k_{0} \sqrt{\epsilon_{h}}, \\
k_{\epsilon_{r}} & =k_{0} \sqrt{\epsilon_{r}}, \\
\lambda_{0} & =\sqrt{\lambda^{2}-k_{0}^{2}}, \\
\lambda_{h} & =\sqrt{\lambda^{2}-k_{h}^{2}}, \\
\lambda_{\epsilon r} & =\sqrt{\lambda^{2}-k_{\epsilon_{r}}^{2}},
\end{aligned}
$$

With this technique, fast convergence for the difference integral can be obtained. As an example, Fig. 2 shows a comparison of three integrands, $A_{m n}, D_{m n}$, and $A_{m n}+D_{m n}$, used in the computation of self impedance of a triangular subdomain function with two joined symmetric $45^{\circ}$ isosceles triangles. It is observed that the convergence of the integral for the imaginary part of $D_{m n}$ is improved by the order of $\lambda^{2}$ when compared to $A_{m n}+D_{m n}$. The real parts of all the integrands, which result from the loss due to radiation, are nonzero for only a finite range. A singularity appears in the imaginary part of the integrand $D_{m n}$. It is due to a surface-wave pole whose residue contribution in the integration represents the surface-wave radiation. In this analysis, we calculate both asymptotic and difference integrals very efficiently. The numerical techniques employed for both integrals are further discussed below.

\section{B. Space-Domain Treatment of the Asymptotic Integral}

The asymptotic integral in (8) represents the mutual impedance between the expansion function $\vec{f}_{n}$ and the testing function $\vec{f}_{m}$ in an infinite homogeneous medium with permittivity $\epsilon_{h}$. It can be expressed in the space domain as

$$
\begin{aligned}
Z_{m n}^{a}= & \int_{T_{m}^{+}+T_{m}} \int_{T_{n}^{+}+T_{n}^{-}} g_{h}\left(\vec{r}, \vec{r}^{\prime}\right) \vec{f}_{m}(\vec{r}) \\
& \cdot \vec{f}_{r^{\prime}}\left(\vec{r}^{\prime}\right) d s^{\prime} d s+\frac{1}{k_{h}^{2}} \\
& \cdot \int_{T_{m}^{+}+T_{m}^{-}} \int_{T_{n}^{+}+T_{\bar{n}}} \vec{f}_{m}(\vec{r}) \\
& \cdot \nabla_{s}\left[\nabla_{s} g_{h}\left(\vec{r}, \vec{r}^{\prime}\right) \vec{f}_{n}\left(\vec{r}^{\prime}\right)\right] d s^{\prime} d s
\end{aligned}
$$

where

$$
g_{h}\left(\vec{r}, \vec{r}^{\prime}\right)=\frac{-j \omega \mu_{0}}{4 \pi} \frac{\exp \left[-j k_{h}\left|\vec{r}-\vec{r}^{\prime}\right|\right]}{\left|\vec{r}-\vec{r}^{\prime}\right|} .
$$

Equation (21) is proportional to the scalar potential Green's function in the same homogeneous medium. $\vec{r}$ and $\vec{r}^{\prime}$ are position vectors of the testing point $(x, y)$ and the source point $\left(x^{\prime}, y^{\prime}\right)$ respectively. It should be noted that $g\left(\vec{r}, \vec{r}^{\prime}\right)$ exhibits a $1 /\left|\vec{r}-\vec{r}^{\prime}\right|$ singularity as $\vec{r} \rightarrow$ $\vec{r}^{\prime}$. With the application of operator $\nabla_{s} \nabla_{s}$ on $g_{h}\left(\vec{r}, \vec{r}^{\prime}\right)$,

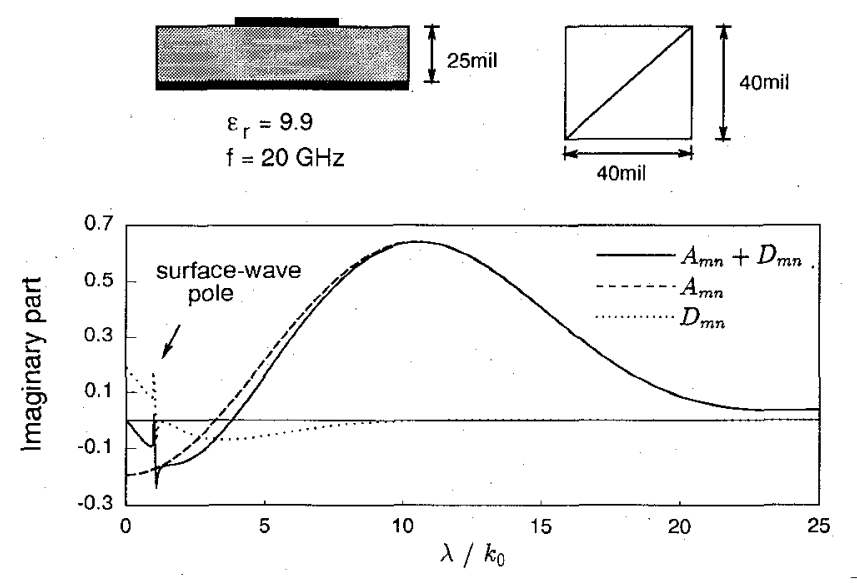

(a)

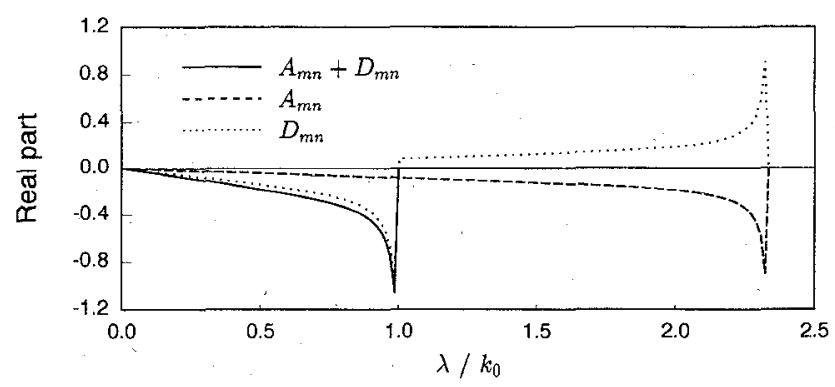

(b)

Fig. 2. Comparison of (a) imaginary part (b) real part of three integrands used in the self impedance calculation, as a function of real $\lambda$.

a higher order singularity appears in the integrand of the second integral of (20) which makes the numerical evaluation of the integral very difficult when the testing points are close to the source points. However, this difficulty can be alleviated if one of the gradients is transferred to act on $\left(x^{\prime}, y^{\prime}\right)$ instead of $(x, y)$. For the scalar potential Green's function in a homogeneous medium, the following identity is obvious:

$$
\nabla_{s} g_{h}\left(\vec{r}, \vec{r}^{\prime}\right)=-\nabla_{s}^{\prime} g_{h}\left(\vec{r}, \vec{r}^{\prime}\right) .
$$

At this point it is convenient to employ a surface vector calculus identity [22] along with the property 1 of $\vec{f}_{p}$. Both gradients on $g_{h}\left(\vec{r}, \vec{r}^{\prime}\right)$ with respect to $(x, y)$ and $\left(x^{\prime}, y^{\prime}\right)$ respectively can be transferred to act on the current expansion functions. As a result, (20) can be rewritten as

$$
\begin{aligned}
Z_{m n}^{a}= & \int_{T_{m+T_{\bar{m}}^{+}}} \int_{T_{n}^{+}+T_{\bar{n}}^{-}} g_{h}\left(\vec{r}, \vec{r}^{\prime}\right)\left[\vec{f}_{m}(\vec{r}) \vec{f}_{n}\left(\vec{r}^{\prime}\right)\right. \\
& \left.-\frac{1}{k_{h}^{2}} \nabla_{s} \cdot \vec{f}_{m}(\vec{r}) \nabla_{s}^{\prime} \cdot \vec{f}_{n}\left(\vec{r}^{\prime}\right)\right] d s^{\prime} d s .
\end{aligned}
$$

In general, the surface divergence of $\vec{f}_{n}$ can be easily derived from (2). It is a constant given as

$$
\nabla_{s} \cdot \vec{f}_{n}(x, y)=\left\{\begin{array}{cc}
\frac{l_{n}}{A_{n}^{+}}, & x, y \text { in } T_{n}^{+} \\
-\frac{l_{n}}{A_{n}^{-}}, & x, y \text { in } T_{n}^{--} \\
0, & \text { otherwise. }
\end{array}\right.
$$


After substituting (24) into (23), numerical computation of (23) is greatly simplified. Rao et al. [15] have developed an efficient method to evaluate this reaction integral using normalized area coordinates. The singularity within $g_{h}\left(\vec{r}, \vec{r}^{\prime}\right)$ can be extracted to yield a smooth function. Then the integration of the integrand using this smooth function over the testing triangle can be approximated well by the value of integrand at the triangle centroid times the area of triangle, while the integration associated with the singular term can be evaluated in closed form [23]. This singularity extraction technique allows the double surface integrals to be reduced to single surface integrals which may be numerically calculated by a quadrature technique appropriate for triangular domains [24].

\section{Spectral-Domain Numerical Evaluation of the Difference Integral}

Due to the rapid decay of $D_{m n}(\lambda)$ as $\lambda \rightarrow \infty$, the infinite integration range of (9) may be truncated to $(0, A)$. The upper limit $A$ must be chosen large enough to satisfy $\overline{\widetilde{G}}$ $\simeq \bar{G}$ for $\lambda>A$, which can result from

$$
\sqrt{A^{2}-k_{0}^{2}} \simeq \sqrt{A_{2}-k_{h}^{2}} \simeq \sqrt{A_{2}-k_{\epsilon r}^{2}} \simeq A
$$

and

$$
\tanh A h \simeq \operatorname{coth} A h \simeq 1 .
$$

From (25) and (26), it can be concluded that the determination of $A$ depends on the dielectric constant and electrical thickness of the substrate. For a smaller dielectric constant or electrically thicker substrates, $A / k_{0}$ may be chosen smaller to reduce numerical expense in evaluation of the difference integral. Moreover, $D_{m n}(\lambda)$ contains all the singularities, namely the surface wave poles, over the $\lambda$ domain. One way of performing the integration from 0 to $A$ is to deform the contour above the real axis where the integrand is well behaved [25]. This method is particularly useful in a multi-layered structure because knowledge of individual pole locations is not required. The evaluation of the difference integral can be considered as the key contribution from the multi-layered structure to each impedance matrix element. It includes the surfacewave radiation and part of the space-wave.

Due to the fact that the difference integral is evaluated in the spectral domain with an integrand equivalent to multiplication of the spectral-domain dyadic Green's function and the Fourier transforms of the basis functions, many advantageous numerical schemes can be exploited. First of all, the Green's function depends on the material parameters such as the dielectric constant and thickness, while the basis functions depend on the microstrip parameters such as microstrip dimension and discontinuities. Therefore, one can consider the programming for the spectral-domain dyadic Green's function and the Fourier transforms of the basis functions as two independent black boxes. Any change in parameters affects only one box without affecting the numerical integration scheme. For instance, we extend our previous work [11] which used a combination of piecewise sinusoidal and pulse functions (rectangular subdomain functions) to model any irregular rectangularly-shaped microstrip discontinuity to the current work using triangular subdomain functions to model arbitrarily-shaped microstrip discontinuities. Since both rectangular and triangular subdomain functions in the space domain correspond to similar, broad and smoothlybehaved spectra in the spectral domain, most of the numerical procedure can be repeated with the exception of substituting a new Fourier transform in place of the rectangular basis functions. Thus, the choice of basis functions in the spectral-domain technique is very flexible. Unlike the spectral-domain method, the spatial convolution of the Green's function and basis functions is intimately related to the shape of the basis function domains. Any change of basis function implies a completely different numerical development in the space domain.

The spectral-domain difference integral has been reduced to a double finite integral with a well-behaved integrand. We may further reduce CPU time by employing some special numerical schemes developed in the spectral domain. These schemes are described as follows.

C.1 Separation of Spectral Variables for the SpectralDomain Dyadic Green's Functions: The difference between the spectral-domain dyadic Green's functions $\overline{\bar{G}}(\lambda$, $\theta)$ and $\bar{G}_{a}(\lambda, \theta)$ may be written as a summation of products of functions which contain one spectral variable only. Thus

$$
\overline{\overline{\tilde{G}}}(\lambda, \theta)-\overline{\overline{\tilde{G}}}_{a}(\lambda, \theta)=\frac{-j \omega \mu_{0}}{k_{0}^{2}}\left\{\overline{\bar{C}} g_{1}(\lambda)+\overline{\bar{\Theta}}(\theta) g_{2}(\lambda)\right\}
$$

where

$$
\begin{aligned}
\overline{\bar{C}} & =\left[\begin{array}{ll}
1 & 0 \\
0 & 1
\end{array}\right], \\
\overline{\bar{\theta}}(\theta) & =\left[\begin{array}{ll}
\cos ^{2} \theta & \cos \theta \sin \theta \\
\cos \theta \sin \theta & \sin ^{2} \theta
\end{array}\right], \\
g_{1}(\lambda) & =\frac{k_{0}^{2}}{\lambda_{0}+\lambda_{\epsilon_{r}} \operatorname{coth} \lambda_{\epsilon r} h}-\frac{k_{0}^{2}}{2 \lambda_{h}}
\end{aligned}
$$

and

$$
\begin{aligned}
g_{2}(\lambda)= & \frac{-\lambda^{2}}{\lambda_{0}+\lambda_{\epsilon_{r}} \operatorname{coth} \lambda_{\epsilon_{r}} h}+\frac{\lambda^{2}}{2 \lambda_{h} \epsilon_{h}} \\
& +\frac{\lambda^{2} \lambda_{0}\left(\epsilon_{r}-1\right)}{\left(\lambda_{0}+\lambda_{\epsilon r} \operatorname{coth} \lambda_{\epsilon_{r}} h\right)\left(\epsilon_{r} \lambda_{0}+\lambda_{\epsilon r} \tanh \lambda_{\epsilon_{r}} h\right)} .
\end{aligned}
$$

From (27)-(31), it can be seen that the mathematically complicated functions $g_{1}$ and $g_{2}$ in $\overline{\bar{G}}-\overline{\bar{G}}_{a}$ are $\theta$ independent and can be left out of the inner integral. Hence, 
the difference integral in (9) may be written as

$$
\begin{aligned}
Z_{m n}^{d}= & \frac{-j \omega \mu_{0}}{4 \pi^{2} k_{0}^{2}} \int_{0}^{A} \lambda g_{1}(\lambda) \int_{0}^{2 \pi}\left[\overline{\bar{C}} \cdot \overrightarrow{\tilde{f}}_{n}(\lambda ; \theta)\right] \\
& \cdot \overrightarrow{\tilde{f}}_{m}^{*}(\lambda, \theta) d \theta d \lambda+\frac{-j \omega \mu_{0}}{4 \pi^{2} k_{0}^{2}} \\
& \cdot \int_{0}^{A} \lambda g_{2}(\lambda) \int_{0}^{2 \pi}\left[\overline{\bar{\theta}}(\theta) \overrightarrow{\tilde{f}}_{n}(\lambda, \theta)\right] \\
& \cdot \overrightarrow{\tilde{f}}_{m}^{*}(\lambda, \theta) d \theta d \lambda .
\end{aligned}
$$

The most numerically intensive calculation (almost $90 \%$ of the CPU time) in computing (32) is the numerical sampling of the integrands of the inner integrations over both $\lambda$ and $\theta$ domains. However, one realizes that upon close inspection, the dyadic functions $\overline{\bar{C}}$ and $\overline{\bar{\theta}}(\theta)$ in these integrands are independent of any material parameters. In addition, the inner integrations in (32) are frequency independent, assuming (25) and (26) are satisfied. Hence, without changing the expansion and testing functions, we can save a great amount of CPU time by computing the inner integrations only once for various material parameters or different frequencies.

C.2 Translation of Basis Functions: In most practical circuit applications, the shape of a microstrip junction remains quite regular such that only a few types of triangular subdomain functions are required to model an entire junction. For instance, Fig. 3 shows a grid structure which can model a microstrip junction whose boundary edge slopes are restricted to $0^{\circ}, 90^{\circ}$ and $\tan ^{-1}\left(s_{y} / s_{x}\right)$. It can be seen that only 3 different geometries of basis functions, $\vec{f}_{p q}^{1}, \vec{f}_{p q}^{2}$, and $\vec{f}_{p q}^{3}$ are required to create the grid. Integer subscripts $p$ and $q$ herein identify the position of each expansion function. The translation relation for the $i$ th type basis function is given as

$$
\vec{f}_{p q}^{i}(x, y)=\vec{f}_{00}^{i}\left(x-p s_{x}, y-q s_{y}\right) .
$$

It is a well-known property that the translation of a function in the space domain represents a phase shift of the function in the spectral domain. Thus

$$
\overrightarrow{\tilde{f}}_{p q}^{i}\left(k_{x}, k_{y}\right)=\overrightarrow{\tilde{f}}_{00}^{i}\left(k_{x}, k_{y}\right) e^{j\left(k_{x} p s_{x}+k_{y} q s_{y}\right)} .
$$

By employing (34) in (32), it can be seen that the difference integral involving the expansion function $\vec{f}_{p^{\prime} q^{\prime}}^{j}$ and testing function $\vec{f}_{p q}^{i}$ depends only on their relative position $(u, v)=\left(p^{\prime}-p, q^{\prime}-q\right)$. In addition, for the difference integrals associated with two different relative position vectors, the integrands are the same except for a phase shift factor. Therefore, it is numerically efficient to compute and store the values of the difference integrals as a function of the relative position indices $u$ and $v$. As a result, a data set for all the possible impedance elements between any $j$ th type expansion functions and $i$ th type testing functions inside the grid is constructed and expressed as

$$
Z_{i j}(u, v)=Z_{i j}^{a}(u, v)+Z_{i j}^{d}(u, v) .
$$

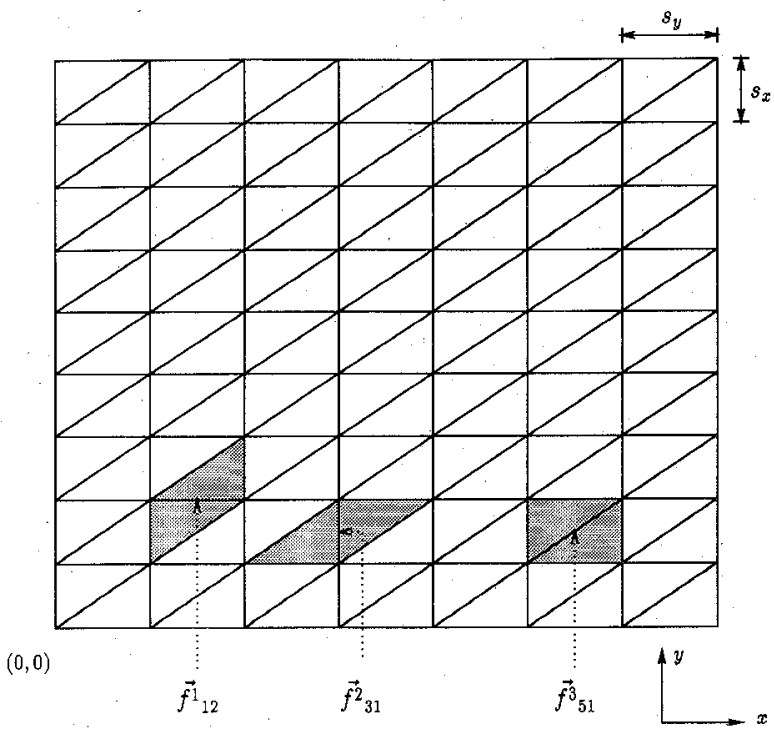

Fig. 3. Grid structure using three different types of triangular subdomain expansion functions.

After employing reciprocity, the following redundancies of impedance elements can be found:

$$
Z_{i j}(u, v)=Z_{j i}(-u,-v)
$$

\section{Evaluation of Radiation ANd Surface-Wave LOSSES}

Since radiation and surface waves are unavoidable physical effects for open microstrip discontinuities, a generalized method to rigorously determine both losses can be developed in the spectral domain [14]. Generally speaking, the method investigates the radiation loss by performing the surface integral of the Poynting vector associated with the radiated space waves in the direction $(\hat{z})$ normal to the substrate. The integration plane is chosen as an infinite plane (denoted by $\Sigma$ ) which is parallel and above the plane of discontinuities. Thus, the surface integral for radiation loss is given as

$$
P_{r a d}=\frac{1}{2} \operatorname{Re} \iint_{\Sigma}\left(\vec{E}_{r}(\vec{r}) \times \vec{H}_{r}^{*}(\vec{r})\right) \cdot \hat{z} d x d y
$$

where $\vec{E}_{r}$ and $\vec{H}_{r}$ denote the radiated electric and magnetic fields in free space, respectively. For the surface-wave loss, with the specific characteristics that surface waves propagate along the surface, the surface-wave loss can be found by integrating the Poynting vector over a cylinder of large radius $\rho$ with height extending from the ground plane to infinity. The surface integral for surface-wave loss is given as

$$
P_{s u r}=\int_{0}^{\infty} \int_{0}^{2 \pi} \frac{1}{2} \operatorname{Re} \sum_{i}\left(\vec{E}_{s i}(\vec{r}) \times \vec{H}_{s i}^{*}(\vec{r})\right) \cdot \hat{\rho} \rho d \phi d z,
$$

where subscript $i$ identifies the substrate or the air region and $\vec{E}_{s i}, \vec{H}_{s i}$ denote the surface wave electric and magnetic fields respectively. From [14], the integration in (37) over 
the $x y$ plane can be converted into an integration over the $k_{x} k_{y}$ plane such that the spectral-domain expressions for all the field components can be utilized. Moreover, the integration in (38) with respect to spatial angular variable $\phi$ can be performed in the $\theta$ domain while the integration with respect to $z$ can be evaluated in closed form. The advantage of using spectral-domain field expressions is that the spectra for both radiated space waves and surface waves are finite. The spectrum for the radiated space waves must satisfy $k_{x}^{2}+k_{y}^{2}<k_{0}^{2}$ which corresponds to a range within a circle of radius $k_{0}$. For the surface waves, the spectrum corresponds to circles with radii equal to the surface-wave poles $\lambda_{p}, p=1,2, \cdots$. Due to the finite spectra, the spectral-domain surface integrals for both losses can be accurately calculated. Additionally, only in the spectral domain may the specific propagating characteristics for both radiated space waves and surface waves be so clearly delineated.

\section{Numerical Results and Discussion}

In this paper, all the numerical computations have been performed on an IBM 9000 mainframe system. As an example, we constructed a grid structure as shown in Fig. 3 for the case $s_{x}=s_{y}=4 \mathrm{mil}$ on an alumina substrate $\left(\epsilon_{r}\right.$ $=9.9$ ) with thickness $h=25 \mathrm{mil}$. Six data bases were set up to represent six different kinds of matrix elements $\left(Z_{i j}, i j=11,12,13,22,23,33\right)$. Each data base contains ten data sets associated with ten sampling frequencies within the range 6 to $24 \mathrm{GHz}$. In each data set, the indices $u, v$ vary from -50 to 50 to generate a grid with $100 \times$ 100 cells. The typical computer time for a data base is approximately 4 minutes. The spectral-domain evaluation of the difference integrals takes about $40 \%$ of the total computer time. The grid structure in Fig. 3, which consists of three different types of triangular subdomain functions, can model any microstrip discontinuity whose boundary is restricted to having at most 3 unique slopes. In general, for more complicated geometrical configurations with $n(n \geq 3)$ different boundary slopes, $(2 n-3)$ different types of triangular elements are required. This implies that $\left(2 n^{2}-5 n+3\right)$ data bases need to be set up. Therefore, for modeling arbitrarily-shaped discontinuities with a large number of boundary slopes, nonuniform triangular elements will be preferred.

\section{A. Open-End and Mitered Open-End Discontinuities}

To demonstrate the numerical accuracy of this analysis, the phase term of the reflection coefficient calculated from an open end (shown in Fig. 4) has been compared with the measurements in [26]. Fig. 5 shows the comparison and convergence as the size of the basis functions reduces. It can be seen that excellent agreement can be achieved for the case $s_{x}=2 \mathrm{mil}$ and $s_{y}=4 \mathrm{mil}$. The difference from the measurements is less than $0.5^{\circ}$ over the frequency range from 6 to $24 \mathrm{GHz}$.

From an equivalent circuit point of view, a microstrip open end may be modeled as an ideal transmission line
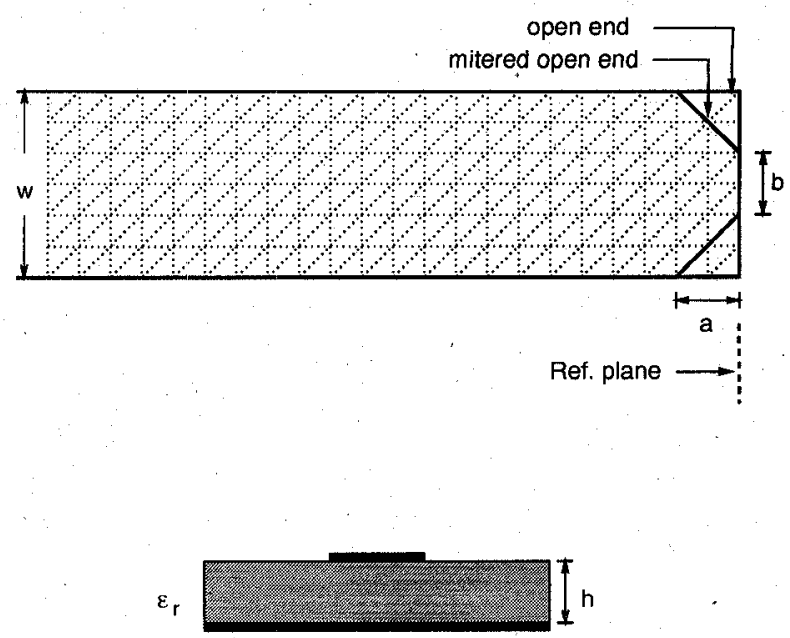

Fig. 4. The geometry of open-end and mitered open-end discontinuities.

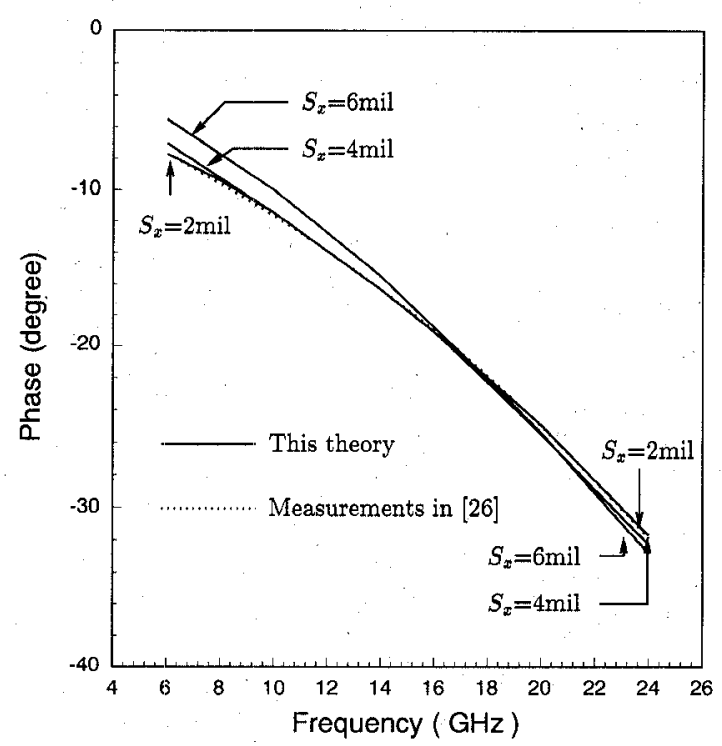

Fig. 5. Phase of $S_{1 \|}$ for an open end $\left(\epsilon_{r}=9.9, w=24 \mathrm{mil}, h=25 \mathrm{mil}\right.$, $\epsilon_{y}=4$ mil).

terminated with a lumped parasitic capacitance. This capacitance may be reduced by properly contouring the conductor in the vicinity of the open end. In this paper, an open end with a simple miter (shown in Fig. 4) is studied. Fig. 6 shows the comparison of two open ends mitered at different angles to the open end without a miter. It can be seen that both open ends with miters are quite successful at reducing the end effects. However, among these three cases, the magnitude of the reflection coefficients calculated is almost the same. In Fig. 7 we plot the ratio of both radiation and surface-wave losses to the incident power as a function of frequency for all three cases. No difference due to geometry can be observed at frequencies up to $24 \mathrm{GHz}$. Fig. 8 shows a typical example of the magnitude of the electric surface currents on a mitered openend discontinuity. These surface current plots demonstrate the edge singularity in the electric current distribution in addition to the typical discretizations associated with the discontinuity in the moment method procedure. 


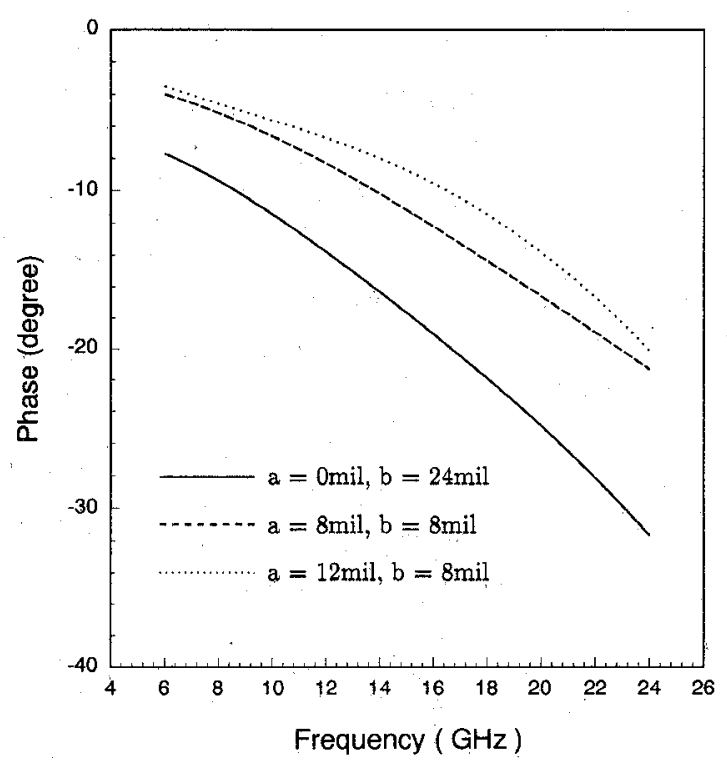

Fig. 6. Phase of $S_{11}$ for an open end with a simple miter $\left(\epsilon_{r}=9.9, w=\right.$ $24 \mathrm{mil}, h=25 \mathrm{mil})$.

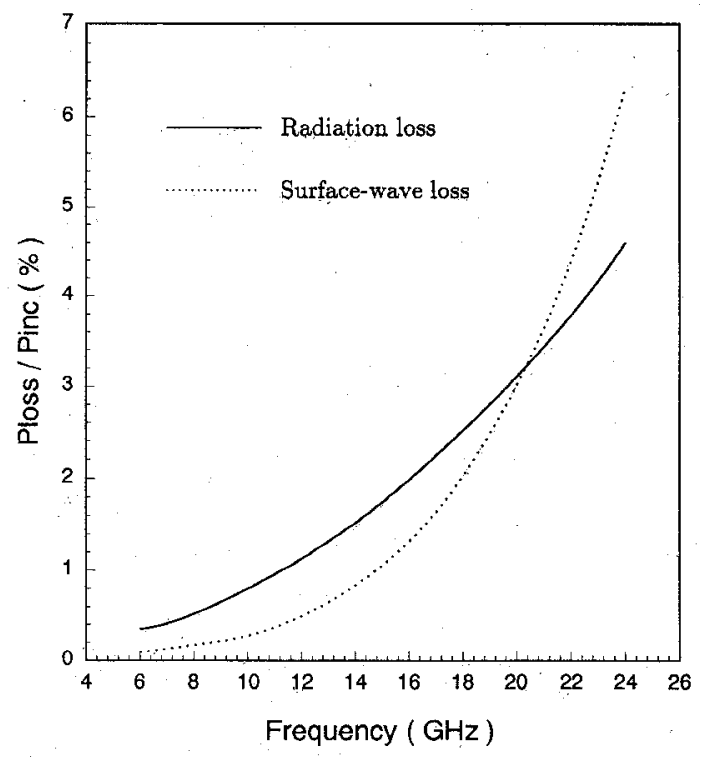

Fig. 7. Power losses versus frequency for all cases in Fig. 6.

\section{B. Right-Angle and Mitered Bend Discontinuities}

The improvements provided by geometrical modification to the outer portion of the right-angle bend with a $45^{\circ}$ miter (shown in Fig. 9) are investigated. Fig. 10 shows the normalized susceptance for both right-angle and mitered bend discontinuities as a function of frequency. As expected, the mitered bend has a smaller susceptance than the right-angle bend over a wide frequency range of interest. Fig. 11 shows the normalized conductance which results from the radiation and surface-wave losses at the junctions. It can be seen that the mitered bend not only reduces the susceptance but also lowers the losses. Fig. 12 shows the normalized electric length for both discontinuities. Comparison of numerical data with measure-
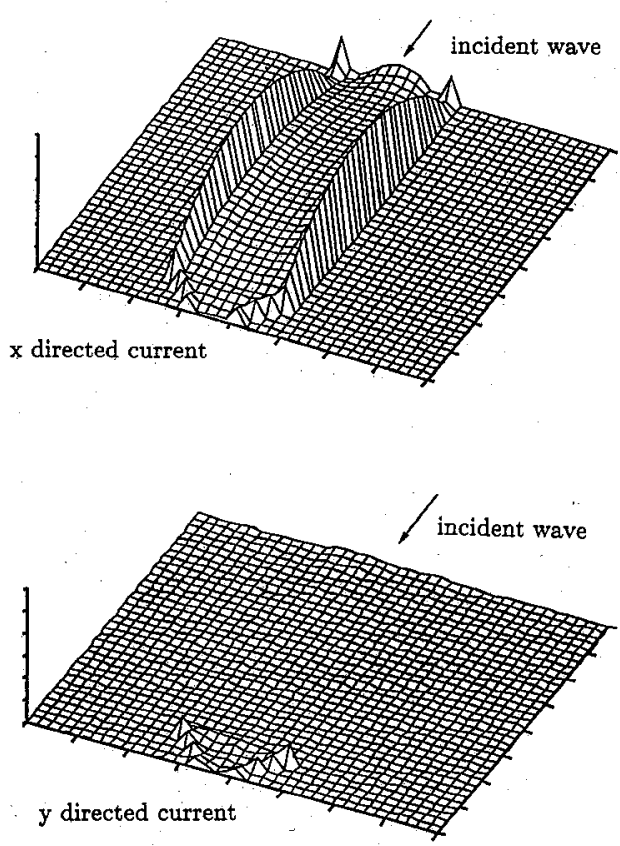

Fig. 8. Magnitude of electric surface currents on a mitered open-end discontinuity $\left(\epsilon_{r}=9.9, w=24 \mathrm{mil}, h=25 \mathrm{mil}, a=8 \mathrm{mil}, b=8 \mathrm{mil}, f=\right.$ $24 \mathrm{GHz})$.

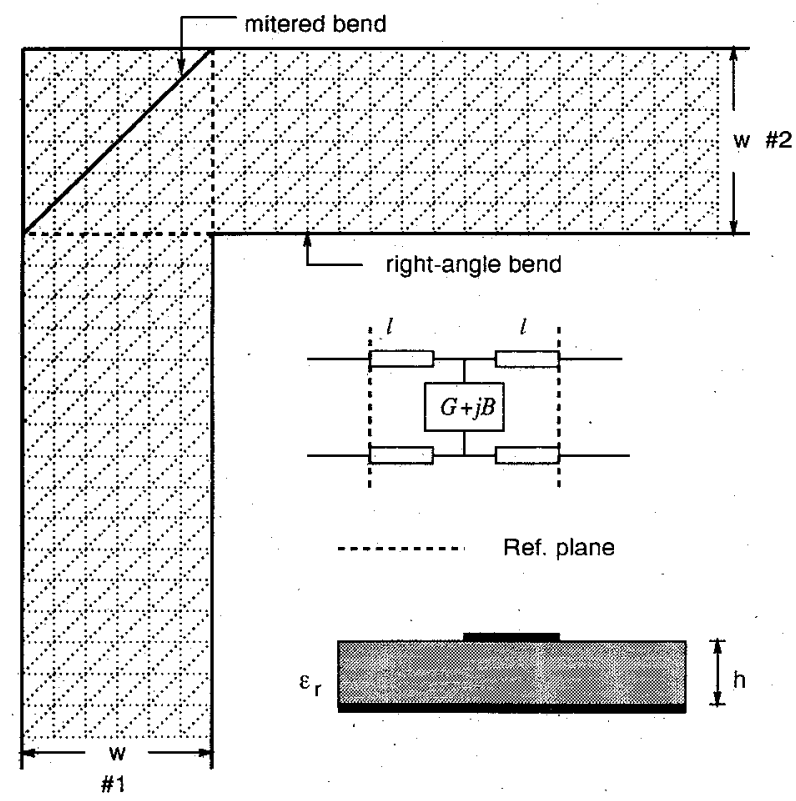

Fig. 9. The geometry of right-angle and mitered bend discontinuities.

ments [27] for susceptance and electric length shows excellent agreement.

Another comparison for both discontinuities on an alumina substrate is shown in Fig. 13. Again, the mitered bend has a smaller reflection coefficient than the rightangle bend. The losses due to radiation and surface waves under the same case are shown in Fig. 14, where we can see that the mitered bend reduces both types of loss. Fig. 15 shows the magnitude of the electric surface currents along with the discretizations for a typical mitered bend discontinuity. 


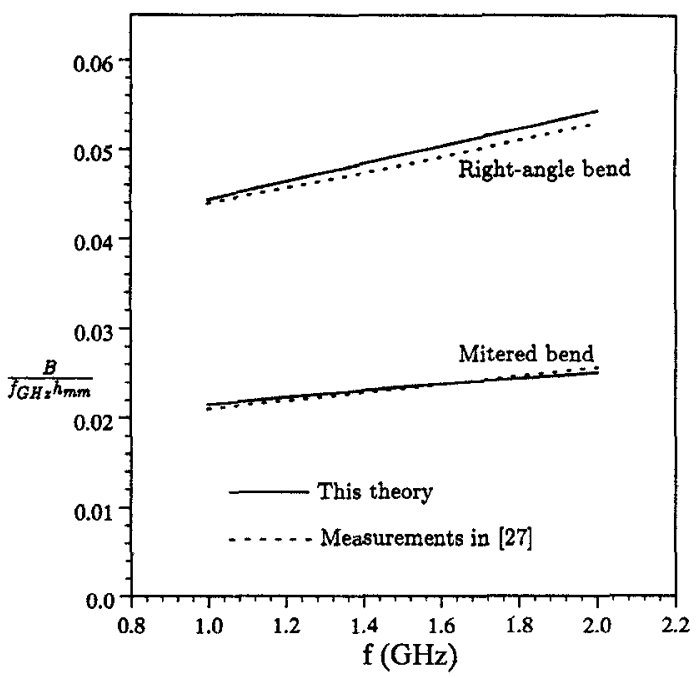

Fig. 10. Frequency dependence of normalized susceptance of right-angle bend and mitered bend discontinuties $\left(\epsilon_{r}=10.8, w=4.572 \mathrm{~mm}, h=\right.$ $5.08 \mathrm{~mm}$ ).

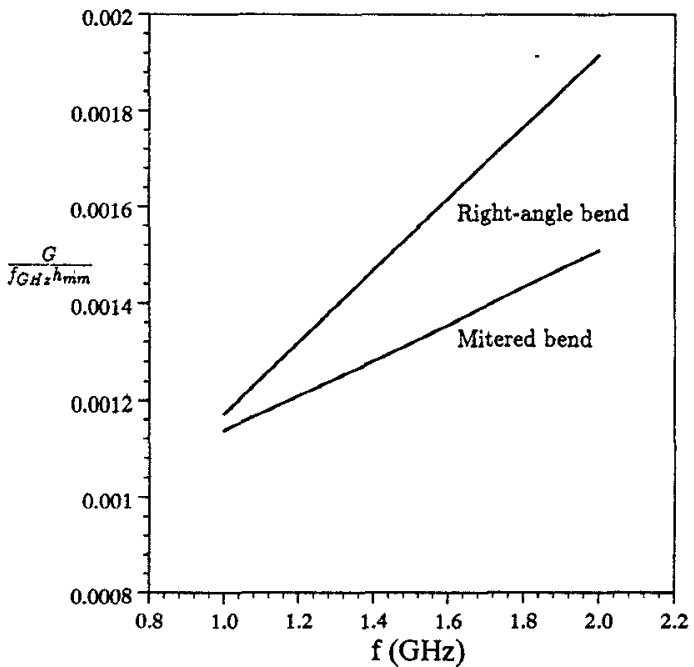

Fig. 11. Frequency dependence of normalized conductance of right-angle bend and mitered bend discontinuities $\left(\epsilon_{r}=10.8, w=4.572 \mathrm{~mm}, h=\right.$ $5.08 \mathrm{~mm}$ )

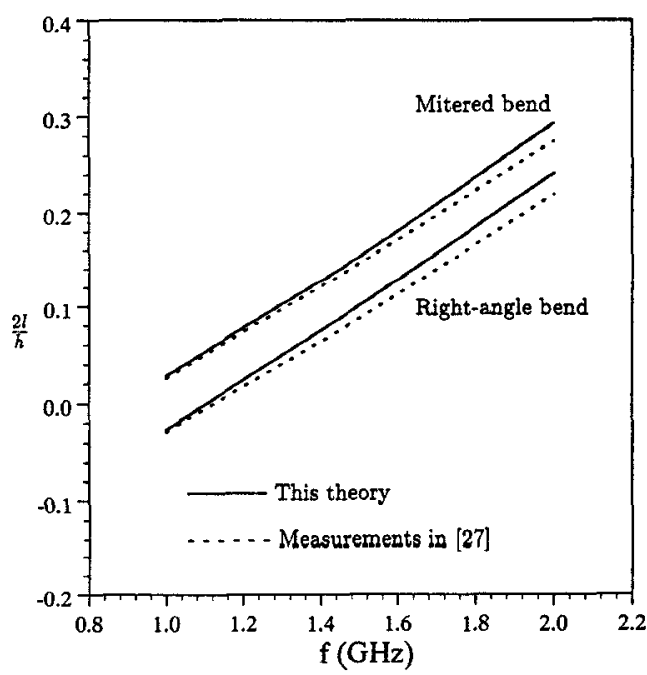

Fig. 12. Frequency dependence of normalized electric length of right-angle bend and mitered bend discontinuities $\left(\epsilon_{r}=10.8, w=4.572 \mathrm{~mm}, h\right.$ $=5.08 \mathrm{~mm}$ ).

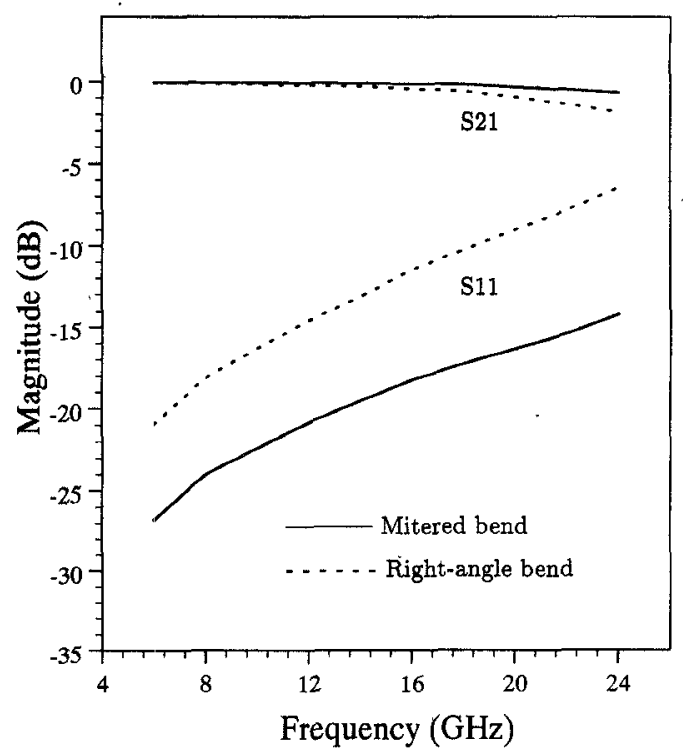

Fig. 13. Magnitude of $S$ parameters of right-angle and mitered bend discontinuities $\left(\epsilon_{r}=9.9, w=24 \mathrm{mil}, h=25 \mathrm{mll}\right)$.

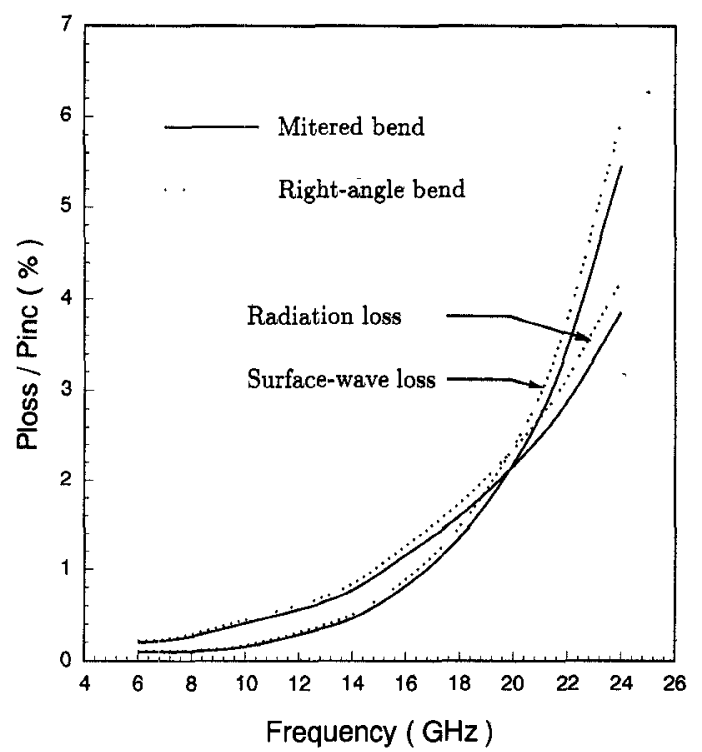

Fig. 14. Power losses versus frequency for right-angle and mitered bend discontinuities.

\section{Basic and Mitered T-Junction Discontinuities}

For compensation of discontinuity reactances of a basic $\mathrm{T}$ junction, the removal of $\mathrm{a} 45^{\circ}$ isosceles triangle is considered. Both discontinuities, basic and mitered $\mathrm{T}$ junctions, are shown in Fig. 16. The magnitude of the scattering parameters for both discontinuities is shown in Fig. 17 , where the comparison with the measured results in [11] for the case of a basic $\mathrm{T}$ junction shows excellent agreement. It is observed that a mitered $\mathrm{T}$ junction has smaller magnitude of $S_{33}$ than a basic T junction. This phenomenon is even more pronounced at higher frequencies. Finally, Fig. 18 shows the magnitude of electric surface currents on a mitered $\mathrm{T}$ junction when the microstripline attached to port \#3 is excited. 

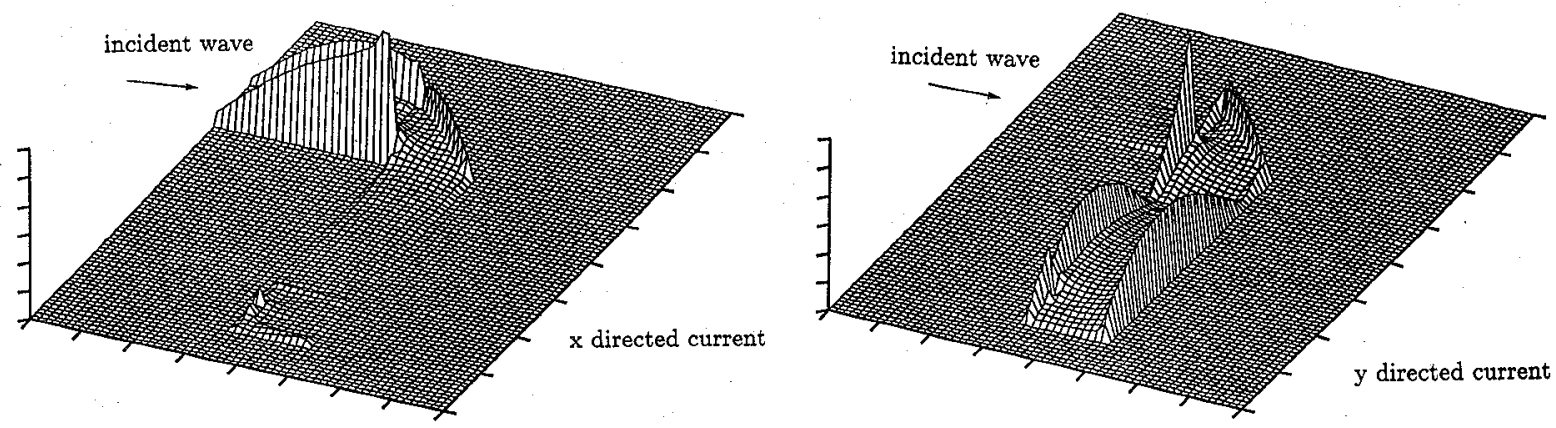

Fig. 15. Magnitude of electric surface currents on a mitered bend discontinuity $\left(\epsilon_{r}=9.9, w=24\right.$ mil, $\left.h=25 \mathrm{mil}, f=24 \mathrm{GHz}\right)$.
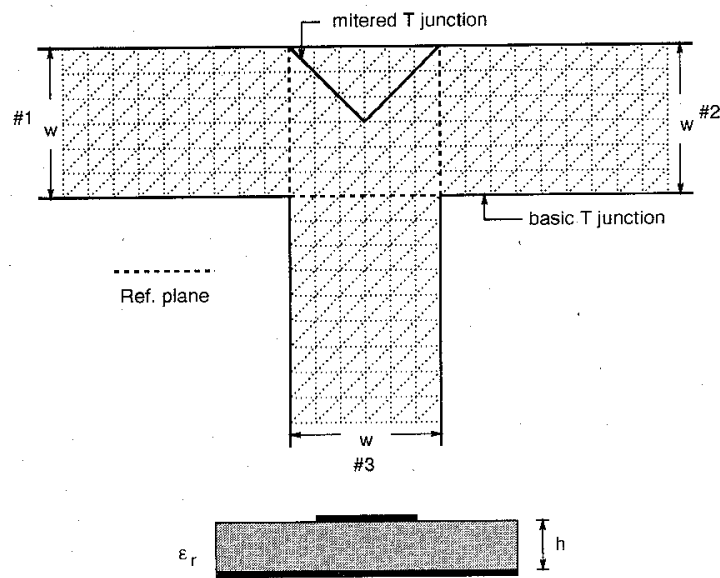

Fig. 16. The geometry of basic and mitered $\mathrm{T}$ junctions.

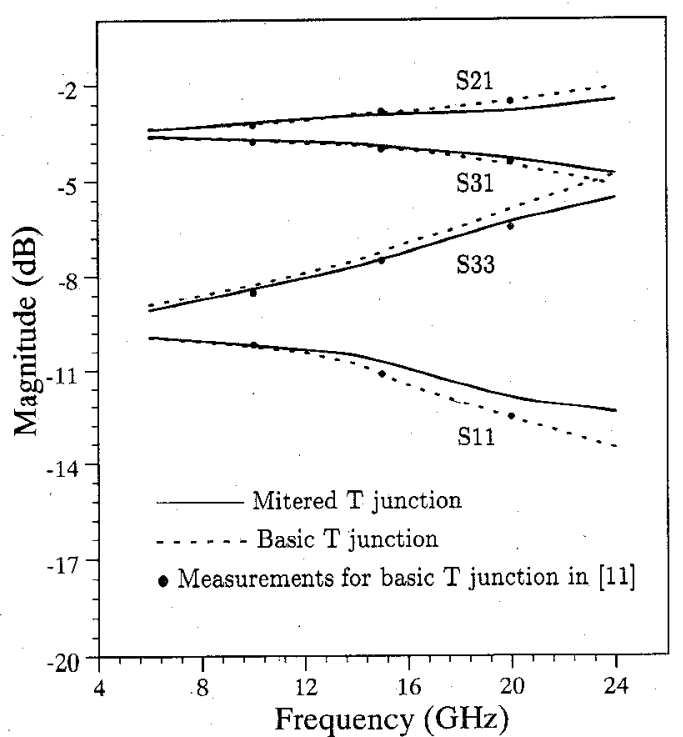

Fig. 17. Magnitude of $S$ parameters of basic and mitered T junctions $(\epsilon$, $=9.9, w=24 \mathrm{mil}, h=25 \mathrm{mil}$ ).

\section{Conclusions}

A full-wave spectral-domain analysis using Galerkin's method with triangular sub-domain basis functions has been found to be a very accurate method to analyze compensated microstrip discontinuities. Several examples were investigated including compensation of open-end,

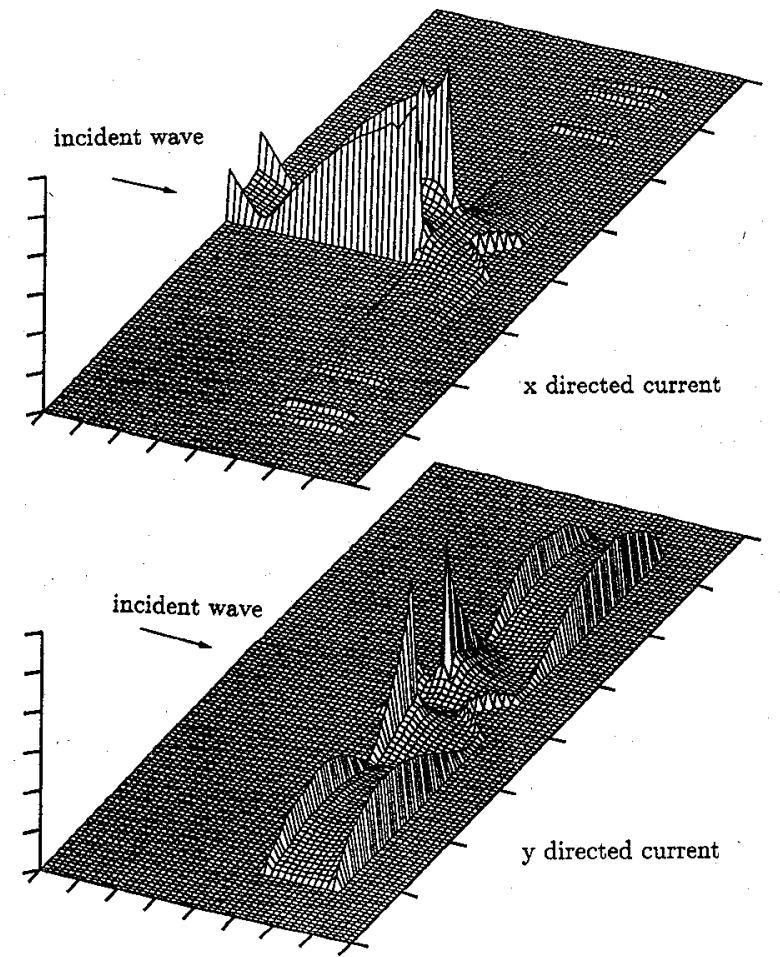

Fig. 18. Magnitude of electric surface currents on a mitered T junction $\left(\epsilon_{r}\right.$ $=9.9, w=24 \mathrm{mil}, h=25 \mathrm{mil}, f=24 \mathrm{GHz}$ ).

bend, and T-junction discontinuities. This work has demonstrated the concept of a grid structure and a data base for storage of impedance matrix elements from which many different geometries may be analyzed.

Radiation and surface-wave losses for the mitered open end and mitered bend were also examined. In the cases studied, mitering has no noticeable effects on the losses associated with an open end, but these losses are slightly reduced for a mitered bend. Calculated $S$ parameters and equivalent circuit values were verified by comparison with available measured data for some cases. These comparisons showed excellent agreement.

\section{REFERENCES}

[1] M. Dydyk, "Master the T-junction and sharpen your MIC designs," Microwaves, vol. 16, no. 5, pp. 184-186, May 1977.

[2] K. G. Gupta and R. Chadha, "Design real-world stripline circuits," Microwaves, vol. 17, no. 12, pp. 70-80, Dec. 1978.

[3] R. Mehran, "Compensation of microstrip bends and Y-junctions with 
arbitrary angle," IEEE Trans. Microwave Theory Tech., vol. MTT26, pp. 400-405, June 1978.

[4] R. Chadha and K. C. Gupta, "Compensation of discontinuities in planar transmission lines," IEEE Trans. Microwave Theory Tech., vol. MTT-30, pp. 2151-2156, Dec. 1982.

[5] P. B. Katehi and N. G. Alexopoulos, "Frequency-dependent characteristics of microstrip discontinuities in millimeter-wave integrated circuits," IEEE Trans. Microwave Theory Tech,, vol. MTT-33, pp. 1029-1035, Oct. 1985.

[6] W. P. Harokopus and P. B. Katehi, "Characterization of microstrip discontinuities on multilayer dielectric substrates including radiation losses," IEEE Trans. Microwave Theory Tech., vol. 37, no. 12, pp. 2058-2066, Dec. 1989.

[7] R. W. Jackson and D. M. Pozar, "Full wave analysis of microstrip open-end and gap discontinuitics," IEEE Trans. Microwave Theory Tech., vol. MTT-33, pp. 1036-1042, Oct. 1985.

[8] H. Y. Yang and N. G. Alexopoulos, "Basic building blocks for high frequency interconnects: Theory and experiment," IEEE Trans. Microwave Theory Tech., vol. 36, pp. 1542-1546, Oct. 1989.

[9] H. Y. Yang and N. G. Alexopoulos, "Microstrip open-end and gap discontinuities in a substrate-superstrate structure," IEEE Trans. Microwave Theory Tech., vol. 37, pp. 1542-1546, Oct. 1989.

[10] R. W. Jackson, "Full-wave, finite element analysis of irregular microstrip discontinuities, "IEEE Trans. Microwave Theory Tech., vol. 37, pp. 81-89, Jan. 1989.

[11] S. C. Wu, H. Y. Yang, N. G. Alexopoulos, and I. Wolff, "A rigorous dispersive characterization of microstrip cross and $\mathbf{T}$ junctions," IEEE Trans. Microwave Theory Tech., vol. 38, pp. 18371844, Dec. 1990.

[12] J. R. Mosig, "A Arbitrarily shaped microstrip structures and their analysis with a mixed potential integral equation,"' IEEE Trans. Microwave Theory Tech., vol. 36, pp. 314-323, Feb. 1988.

[13] K. A. Michalski and D. Zheng, "Analysis of microstrip resonators of arbitrary shape," IEEE Trans. Microwave Theory Tech., vol. 40. pp. 112-119, Jan. 1992.

[14] T. S. Horng, S. C. Wu, H. Y. Yang, and N. G. Alexopoulos, "A generalized method for distinguishing between radiation and surfacewave losses in microstrip discontinuities," IEEE Trans. Microwave Theory Tech., vol. 38, pp. 1800-1807, Dec. 1990.

[15] S. M. Rao, D. R. Wilton, and A. W. Glisson, "Electromagnetic scattering by surfaces of arbitrary shape," IEEE Trans. Antennas Propagat., vol. AP-30, pp. 409-418, May 1982.

[16] D. M. Pozar, "Improved computational efficiency for the method of moments solution of printed dipoles and patch," Electromagnetics, vol. 3, pp. 299-309, 1983.

[17] D. R. Jackson and N. G. Alexopoulos, "An asymptotic extraction technique for evaluating Sommerfeld-type integrals," IEEE Trans. Antennas Propagat., vol. AP-34, pp. 1467-1470, Dec. 1986.

[18] T. Itoh, "Spectral domain immitance approach for dispersion characteristics of generalized printed transmission lines," IEEE Trans. Microwave Theory Tech., vol. MTT-28, pp. 733-736, July 1980.

[19] B. Houshmand, W. C. Chew and S. W. Lee, "Fourier transform of a linear distribution with triangular support and its applications in electromagnetics," IEEE Trans. Antennas Propagat., vol. AP-39, pp. 252-254, Feb. 1991.

[20] K. McInturff and P. S. Simon, "The Fourier transform of linearly varying functions with polygonal support," IEEE Trans. Antennas Propagat., vol. 39, pp. 1441-1443, Sept. 1991.

[21] W. A. Johnson, D. R. Wilton, and R. M. Sharpe, "Modeling scattering from and radiation by arbitrary shaped objects with the electric field integral equation triangular surface patch code," Electromag netics, vol. 10, pp. 41-63, 1990.

[22] J. Van Bladel, Electromagnetic Fields. New York: McGraw Hill, 1964 , pp. 503 , no. 45.
[23] D. R. Wilton et al., "Potential integrals for uniform and linear source distributions on polygonal and polyhedral domains," IEEE Trans. Antennas Propagat., vol. AP-32, pp. 276-281, Mar. 1984.

[24] P. C. Hammer, O. J. Marlowe and A. H. Stroud, "Numerical integration over simplexes and cones," Mathematical Tables and Other Aids to Computation, vol. 10, pp. 130-137, 1956.

[25] E. H. Newman and D. Forrai, "Scattering from a microstrip patch," IEEE Trans. Antennas Propagat, vol. AP-35, pp. 245-251, Mar. 1987.

[26] G. Gronau and I. Wolff, "A simple broad-band device de-embedding method using an automatic network analyzer with time-domain option,"' IEEE Trans. Microwave Theory Tèch., vol. 37, pp. 479-483. Mar. 1989

[27] R. J. P. Douville and D. S. James, "Experimental study of symmetric microstrip bends and their compensation," IEEE Trans. Microwave Theory Tech., vol. MTT-26, pp. 175-182, Mar. 1978.

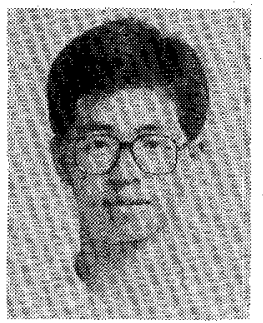

Tzyy-Sheng Horng (S'88-M'92) was born in Taichung, Taiwan on December 7, 1963. He received the B.S.E.E. degree from the National Taiwan University in 1985 and the M.S.E.E. and $\mathrm{Ph} . \mathrm{D}$. degrees from the University of California, Los Angeles, in 1990 and 1992, respectively. From 1985 to 1987, he served in the R.O.C. Air Force as an antiaircraft artillery officer

In September 1992 he joined the faculty of the Electrical Engineering Department at the National Sun Yat-Sen University, Kaohsiung, Taiwan, R.O.C., as an Associate Professor. His research interests include microstrip array antennas and millimeter-wave devices.

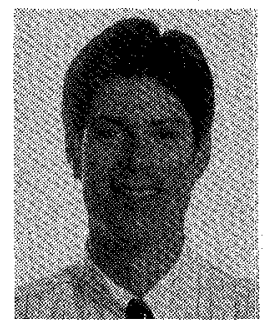

William E. McKinzie received the B.S.E.E. degree from the University of Missouri, Rolla in 1982, and the M.S.E.E. and Ph.D. degrees from the University of California at Los Angeles in 1989 and 1992

From June 1982 to March 1986 he was employed at Motorola GEG in Scottsdale, Arizona, where he worked as an RF circuit designer. From March 1986 to August 1986 he was with MA COM Omni-Spectra in Tempe, Arizona. Dr: McKinzie was a Chancellor's Fellow at UCLA in 1986-87. He worked as a research assistant in the Electrical Engineering Department at UCLA from June 1987 to June 1992. His work included numerical modeling of electromagnetic fields in planar printed apertures of arbitrary shape with applications to circuits and antennas. During this period he received an MTT Society Fellowship in 1989-90.

In July, 1992, he joined the B2 Division of Northrop where his interests include the numerical modeling of three-dimensional structures for antenna and RCS applications.

Nicolaos G. Alexopoulos (S'68-M'69-SM'82-F'87), photograph and biography not available at the time of publication. 